REVUE DE L'INSTITUT

FRANÇAIS D'HISTOIRE

EN ALLEMAGNE

\section{Revue de l'IFHA}

Revue de l'Institut français d'histoire en Allemagne

$2 \mid 2010$

IFHA 2

\title{
Le silence des sources en histoire / Das Schweigen der Quellen in der Geschichtswissenschaft
}

Journée d'études doctorales franco-allemande, Francfort-sur-le-Main, 20 novembre 2009

\section{Guillaume Garner et Thomas Lienhard}

\section{OpenEdition}

\section{Journals}

Édition électronique

URL : http://journals.openedition.org/ifha/225

DOI : $10.4000 /$ ifha. 225

ISSN : 2198-8943

Éditeur

IFRA - Institut franco-allemand (sciences historiques et sociales)

Édition imprimée

Date de publication : 1 janvier 2010

Pagination : 54-62

ISSN : 2190-0078

Référence électronique

Guillaume Garner et Thomas Lienhard, «Le silence des sources en histoire / Das Schweigen der Quellen in der Geschichtswissenschaft », Revue de l'IFHA [En ligne], 2 | 2010, mis en ligne le 01 février 2013, consulté le 15 septembre 2020. URL : http://journals.openedition.org/ifha/225

Ce document a été généré automatiquement le 15 septembre 2020

(CIFHA 


\section{Le silence des sources en histoire / Das Schweigen der Quellen in der Geschichtswissenschaft}

Journée d'études doctorales franco-allemande, Francfort-sur-le-Main, 20 novembre 2009

Guillaume Garner et Thomas Lienhard

\section{NOTE DE L'ÉDITEUR}

Rapport établi par Guillaume Garner et Thomas Lienhard

1 C'est une donnée constante dans la pratique des historiens, et pourtant elle pose des problèmes de méthode sans cesse renouvelés: des pans entiers de l'histoire de l'humanité sont passés sous silence par nos sources. Pour prendre un exemple parmi d'autres, on sait que le discours ecclésiastique du haut Moyen Âge a minimisé ou complètement occulté les phénomènes sociaux qui relevaient du scepticisme religieux, du pouvoir laïc en ville, de l'identité ethnique, de la stratégie d'accumulation de richesses ou encore des relations sexuelles. Mais des remarques analogues pourraient également être appliquées, mutatis mutandis, au discours colonial ou à celui des auteurs masculins sur les femmes, et finalement à tous les domaines de la vie sociale à travers les âges.

2 Les origines de ces lacunes sont multiples, et ce n'était pas le moindre intérêt attendu de cette journée d'études que d'en démêler les causes possibles. Il semble ainsi évident que certains dossiers ne sont pas parvenus jusqu'à nous parce qu'ils étaient classés dans le registre du secret, par exemple dans le domaine financier, technologique ou diplomatique : les acteurs de l'affaire ont alors mis un soin jaloux à en cacher la teneur à leurs contemporains, et par contrecoup à l'historien. Dans d'autres cas, l'omission était clairement liée à un discours dominant qui renâclait à mettre en lumière la vie sociale des dominés, tantôt par simple dédain (parfois inconscient), tantôt dans un 
souci délibéré et plus intéressé de damnatio memoriae. À l'inverse, certaines structures sociales sont sous-représentées dans nos sources non pas parce qu'elles ont fait l'objet d'un refoulement, mais au contraire parce qu'elles étaient admises et connues par tous, n'impliquant donc pas la nécessité d'une rédaction documentaire : tel est le cas des données administratives (notamment fiscales ou juridiques) qui, dans bien des sociétés, ne sont devenues accessibles pour le chercheur que lorsqu'un conflit a donné lieu à l'élaboration d'un texte polémique, alors que les relations sociales normales sont restées immergées dans cet iceberg historique. Enfin, dans un dernier cas de figure, le silence dont souffre l'historien est dû à des facteurs plus techniques : tel est le cas des sociétés sans écriture ou des transmissions textuelles défaillantes, où des données bien connues en une époque déterminée n'ont pas été transmises jusqu'à nous. Les modernistes, par exemple, sont bien en peine de travailler sur la politique économique de la monarchie d'Ancien Régime, les archives du Contrôle général des finances ayant été détruites pendant la Révolution; la même question se pose à propos des archives allemandes détruites pendant la Seconde Guerre mondiale, avec des répercussions méthodologiques bien concrètes, comme la nécessité de recourir à des sources publiées ou à des études réalisées avant la guerre. Les facteurs de silence sont donc aussi puissants que divers.

3 Face à ces vastes zones d'ombre, l'historien se voit confronté à un dilemme. D'une part, étant donnée l'intimité de la relation qui l'unit à ses sources, il trouvera périlleux d'explorer des thèmes qui sont absents de sa documentation : les risques de spéculation et d'arguments a silentio sont bien réels, et dans bien des situations, la sagesse contraint donc à considérer comme perdus les phénomènes qui n'ont pas surnagé dans le flot de la transmission documentaire à travers les âges. Mais d'autre part, il reste parfois nécessaire de prendre en compte l'existence de phénomènes historiques aujourd'hui mal attestés, d'abord parce que certains d'entre eux, même s'ils sont tus par les sources, semblent faire partie du fonctionnement normal de toute société (qui irait postuler l'existence d'un grand groupe humain totalement dépourvu de scepticisme religieux? de réflexion sur les pratiques sexuelles?), et aussi parce que, dans certains cas, des indices ténus dans nos sources contredisent un silence dominant, interdisant au moins à l'historien de considérer, comme cela fut parfois fait en l'absence d'un dossier documentaire important, que tel ou tel phénomène historique n'a tout simplement pas existé. Il arrive ainsi que des données historiques crient malgré le silence : cette contradiction pose des problèmes de méthode intéressants pour l'historien en général, pour le doctorant tout particulièrement.

Telles furent les considérations générales qui avaient présidé à la préparation de cette journée d'études. Celle-ci fut élaborée avec d'autant plus de soin qu'elle constituait la première manifestation scientifique proposée par l'IFHA dans les propres murs de ce dernier. Les organisateurs furent donc d'autant plus heureux de constater que la formule des journées d'études doctorales franco-allemandes, expérimentée bien des fois par la MHFA, n'avait rien perdu de son efficacité. Non seulement le nombre des doctorants intéressés fut tel, à la fois parmi les Allemands et les Français, qu'il fallut se résoudre à une sélection sévère, mais la teneur des communications répondit parfaitement au triple objectif de ce type de manifestation : donner le dernier état de la recherche sur les sujets particuliers, proposer une analyse globale (et francoallemande) sur le sujet général de la rencontre, et permettre à des chercheurs 
confirmés de transmettre leur expérience à des doctorants, en particulier sur des problèmes méthodologiques.

5 Après les remarques introductives de Thomas Lienhard (IFHA), qui reprirent en grande partie les données générales que l'on vient de présenter, la série des communications fut inaugurée par Joachim Henning (Goethe-Universität Frankfurt am Main). Étant donné le sujet de la rencontre, la communication d'un archéologue était particulièrement attendue, et J.Henning se tira magistralement de l'exercice toujours délicat qui consiste à établir des ponts entre archéologues et historiens ( WWege der modernen Archäologie, das ‘ Schweigen der Quellen > zu überwinden »). Le problème spécifique auquel il consacra son intervention fut celui de la raréfaction brutale des tombes identifiées par les archéologues dans certaines régions de l'Austrasie au courant du $\mathrm{V}^{\mathrm{e}}$ siècle; ce silence démographique semble confirmé par une Vie de saint qui décrit un désert en cet espace à la même époque, et par le mutisme complet des autres sources à propos d'une quelconque activité humaine dans ce même contexte. Faut-il en conclure, comme l'ont souvent fait les historiens, à un déclin démographique et économique de cette région à la fin de l'Antiquité, éventuellement en conséquence des invasions barbares?

6 Pour répondre à cette question, l'archéologue commença par présenter une panoplie des techniques récentes auxquelles ses collègues peuvent recourir pour sonder la densité démographique d'un terrain médiéval. Outre l'analyse du Carbone 14 et la dendrochronologie, désormais bien connues par les historiens, il rappela qu'il importait d'évaluer l'évolution de l'érosion pour déterminer si celle-ci, à la période concernée, avait pu affecter la visibilité des tombes recherchées par l'archéologie. Il recourut également aux comparaisons internationales entre des sites archéologiques de la même époque; et en ce domaine, son laboratoire de Francfort est particulièrement bien informé, puisqu'il est impliqué dans plusieurs chantiers d'Amérique du Sud ou d'Europe centrale, permettant ainsi à J. Henning de déceler par exemple des similitudes entre l'Austrasie et Tornow (Slovaquie) que personne n'avait été en mesure d'identifier avant lui. Le conférencier rappela enfin que la visibilité des tombes était en grande partie indépendante du facteur démographique à la fin de l'Antiquité, parce que la raréfaction des dépôts funéraires, due à des évolutions religieuses, rendait les tombes moins faciles à découvrir pour l'archéologue. Compte tenu de tous ces correctifs, il put sortir de l'ombre, à l'encontre de toutes les sources écrites, un site de forte activité économique et religieuse en plein cœur de la zone que les historiens, en l'absence de données archéologiques, considéraient comme vide: il s'agit du site de Tarquimpol (Moselle), probablement identique au chef-lieu de la cité de Decempagus mentionnée par les sources antiques. L'archéologie avait ici permis non seulement de compenser une lacune des textes écrits, mais également de comprendre les motifs des silences affichés par ces derniers : l'auteur de la Vie de saint évoquée plus haut, comme souvent, avait ses raisons propres pour présenter comme désert le lieu d'activité du personnage qu'il souhaitait mettre en valeur.

7 Les difficultés rencontrées par les historiens de la justice pénale et de l'emprisonnement à l'époque moderne firent l'objet de la deuxième intervention prononcée par Falk Bretschneider (EHESS), à partir du cas des prisons saxonnes du $\mathrm{XVII}^{\mathrm{e}}$ au XIX ${ }^{\mathrm{e}}$ siècle ("Stille hinter dicken Mauern? Quellen zur frühmodernen Welt der Einsperrung »). Il y a en effet contraste entre une surabondance de sources normatives et administratives et l'absence de documents provenant des prisonniers, qui apparaissent d'autant plus comme des objets de l'appareil judiciaire et pénal. Il s'agit 
donc de mettre en œuvre des stratégies de lecture de ces sources permettant d'avoir un accès indirect à ces prisonniers. Dans un premier temps, il convient de rappeler que ces sources normatives ne sont évidemment pas un reflet de la réalité, mais qu'elles s'insèrent dans des dispositifs administratifs qui recherchent la stabilisation institutionnelle de cette réalité quotidienne des prisons. Il n'y a donc pas d'opposition entre normes et réalités, puisque ces normes sont précisément des éléments constitutifs de cette réalité dans laquelle elles produisent des effets. Dans un second temps, ces textes normatifs peuvent être confrontés à d'autres sources, notamment les avis, rapports, éléments de correspondance administrative qui font apparaitre les infractions commises contre ces normes. Les prisonniers ne s'expriment certes pas directement dans ces documents, dans la mesure où les membres de l'appareil pénitentiaire s'intéressent moins aux motifs de leurs actions qu'au désordre produit par celles-ci. Mais ces rapports n'en permettent pas moins d'approcher en creux les stratégies de survie des prisonniers, la manière dont ils utilisent les marges d'action que leur laissent les structures d'emprisonnement: il est en particulier possible de retracer les phénomènes de communication, d'interaction entre prisonniers et geôliers qui font de la prison un champ de forces dynamiques et non pas simplement une instance de répression. Ces documents font ainsi apparaître l'obéissance non comme une réalité quotidienne, mais comme une construction bureaucratique, qui ne masque pas l'utilisation de techniques de pouvoir molles. En conclusion de sa présentation, F. Bretschneider considéra que l'expression de "silence des sources" est trompeuse : les sources ne "parlent » pas d'elles-mêmes, et c'est à l'historien qu'il revient de sortir de son mutisme face à elles.

8 L'intervenant suivant fut Nicolas Offenstadt (Université Paris I), qui réussit la prouesse de faire intervenir le thème du cri pour une journée d'études consacrée au silence («Quand le crieur arrive sur la place publique... Jusqu'où le médiéviste peut-il faire parler son époque?»). Il est vrai que le personnage du crieur public, fréquemment représenté dans les sources du bas Moyen Âge mais rarement pris en compte par les historiens, constitue un élément central dans la culture politique de cette période : le porteur de cette charge exerçait un effet non seulement un rôle officiel, mais même une fonction législative puisqu'une loi n'entrait en vigueur que lorsqu'elle avait été proclamée en public. Cette situation pose la question de l'espace public, que Habermas considère comme inexistant pour la période pré-moderne : si l'on se concentre sur le crieur, celui-ci ne constituait-il qu'une courroie de transmission des décisions princières auxquelles la population ne pouvait qu'acquiescer, ou bien sa prestation ouvrait-elle déjà la voie à une discussion et à l'expression d'une opinion publique ?

Pour répondre à cette question, la documentation est relativement aride, et le thème abordé par N. Offenstadt fut peut-être celui pour lequel le silence des sources se montra le plus obstiné au cours de cette journée. Certes, la figure du crieur public est évoquée à la fois dans les sources normatives et dans les textes administratifs urbains; mais ces écrits sont souvent fort peu diserts sur le porteur de cette fonction, et lorsqu'ils décrivent le déroulement de telle ou telle proclamation, la description du public est généralement standardisée et se borne à relever la bonne circulation de l'information nécessaire pour l'entrée en vigueur de la loi. Toutefois, il fut possible à l'orateur de lever ce voile de manière fugace. En particulier, le recours à la documentation iconographique permit de deviner les réactions, parfois très hétérogènes, du public ; l'analyse géographique de l'itinéraire suivi par le crieur constitua un indice supplémentaire pour considérer que, dans ce processus de diffusion, c'était le public 
qui, par un geste actif, se rendait vers l'événement politique au même titre que, dans l'autre sens, le roi allait au peuple; enfin, ces lueurs fugitives furent renforcées par les sources les plus tardives, dans la mesure où à l'extrême fin du Moyen Âge, la documentation devient plus détaillée à propos du crieur. C'était ainsi la chape de plomb d'un silence imposé par en haut qui put être partiellement levée par cette communication.

La seconde demi-journée s'ouvrit sur une intervention de Marian Füssel (Université de Göttingen) consacrée à la question de la violence guerrière au XVIII ${ }^{\mathrm{e}}$ siècle («Spuren der Gewalt. Zur Quellenproblematik einer Kulturgeschichte der Schlacht im 18. Jahrhundert ").Parler de silence des sources à ce sujet est de prime abord paradoxal, tant la bataille est omniprésente dans l'histoire politique et militaire traditionnelle. C. Ginzburg a cependant souligné que ce caractère d'évidence de la bataille est à interroger dans la mesure où la bataille est invisible dans sa globalité pour ceux qui la vivent, en même temps qu'elle est le moment d'un déchaînement de violence, dont les traces dans les sources sont à examiner. Précisément, l'étude de la violence pendant la guerre de Sept Ans se heurte à un silence des sources aux origines diverses. D'une part, les bombardements de la Seconde Guerre mondiale ont occasionné des destructions massives de sources, en particulier à Berlin et à Potsdam. D'autre part, Frédéric II avait interdit la publication de récits relatifs à cette guerre, si bien que l'histoire de ce conflit n'a connu un essor qu'après sa mort en 1786, à une époque où les témoins oculaires étaient peu nombreux, ceux-ci (majoritairement issus de l'état-major) fournissant des récits subjectifs et biaisés. Les récits de soldats, recherchés par les historiens dès la fin $\mathrm{du} \mathrm{XVIII}^{\mathrm{e}}$ siècle, sont donc très rares. Ceux-ci eurent donc recours à des questionnaires adressés aux soldats ou à leurs descendants, ainsi qu'à l'étude d'objets associés aux batailles, au risque de n'obtenir que des informations anecdotiques. M. Füssel évoqua la tentative de Thomas Carlyle de visiter les sites des champs de bataille pour contourner ce silence, tentative qui échoua car la topographie de ces « lieux de mémoire » avait été tellement modifiée que ces sites avaient disparu, rendant impossible tout rapprochement avec les descriptions qu'en donnaient les récits historiques. L'historien de la violence guerrière est donc confronté à un silence dont il peut tirer profit en s'intéressant à la mémoire collective et à la mémoire culturelle de la guerre, en étudiant notamment les modalités de passage entre la première et la seconde de ces formes de mémoire.

11 Étant donné le thème général de la rencontre, on ne pouvait faire l'impasse sur la question des deperdita documentaires. Tel fut le sens de la contribution, au titre provocateur, de Marc Smith (École Nationale des Chartes, «La paléographie des écrits disparus »). L'orateur explora le champ des textes qui avaient soit entièrement disparu, soit avaient été négligés par l'historiographie parce qu'ils n'étaient conservés que sous des formes minoritaires d'écriture ou de support matériel: furent ainsi évoquées notamment les tablettes de cire, les écorces de bouleaux ou encore les tablettes vandales d'encre sur bois. Présentant l'historiographie de ce champ de recherche, M. Smith rappela que celui-ci n'avait d'abord été exploré que de manière auxiliaire, pour "boucher les trous» dans l'évolution générale des écritures que la paléographie cherchait à reconstituer. Mais progressivement, un certain nombre de facteurs pondérateurs, et notamment une réflexion à propos des usages pratiques de tel ou tel type d'écriture qui semblait marginal au premier abord, avait incité les connaisseurs à accorder une attention plus spécifique à ce type de documentation. En particulier, 
l'invention de la paléographie dynamique par Jean Mallon avait largement contribué à réhabiliter le rôle de ces textes dans l'histoire de l'écriture.

Ces divers facteurs de réévaluation donnèrent lieu, dans les dernières décennies, à des bouleversements considérables dans notre connaissance de l'écrit à travers les âges. On savait déjà depuis Maffei que l'écriture romaine, pourtant peu représentée dans les manuscrits du haut Moyen Âge, avait influencé les écritures dites «nationales » bien plus que ne l'avait fait l'apport culturel des hypothétiques invasions barbares. Mais M. Smith se livra également à une belle réhabilitation d'autres documents, en particulier des tablettes de cire : même si les textes véhiculés par ces supports furent rarement conservés parce qu'effacés ou brûlés, l'orateur put cependant démontrer qu'ils avaient été à la fois plus abondants que d'autres et plus influents pour l'évolution de l'écriture, et que ce type de support, à cheval sur l'époque du papyrus, du parchemin et du papier, avait été le plus durable dans l'histoire de l'écrit en Occident. Cette présentation fut ainsi à la fois un modèle de pédagogie, une leçon de modestie face à la masse des textes disparus et une convaincante réévaluation du rôle important joué par des formes de textes souvent négligées lorsqu'elles ont été conservées.

La journée se clôtura par la communication de Paul Pasteur (Université de Rouen), consacrée au champ, en pleine expansion depuis une trentaine d'années, de l'histoire du genre ( Des silences des femmes à ceux sur les hommes à l'époque contemporaine »). P. Pasteur opéra dans un premier temps un retour en arrière sur l'histoire des femmes, souvent promue par des historiennes qui étaient proches des courants féministes et qui avaient préalablement travaillé sur les ouvriers ou les déviants. Se posait alors en particulier la question de la manière dont l'historien peut avoir accès aux femmes des milieux populaires, à une époque où prévalait l'impression que celles-ci étaient absentes des sources. Les problématiques se sont quelque peu infléchies avec l'essor des réflexions sur la notion de genre (gender) à partir du milieu des années 1980. En faisant du genre une construction, une manière de vivre des différences naturelles, ces travaux reprenaient les réflexions de Simone de Beauvoir en insistant sur la distinction entre sexe, genre et sexualité. Ces approches ont d'une part suscité un mouvement de recherche et d'exhumation de sources produites par les femmes, et qui étaient restées longtemps inutilisées, comme en témoignent les séries d'écrits rédigés par des femmes proches de la social-démocratie autrichienne à la fin du $\mathrm{XIX}^{\mathrm{e}}$ et au début du XX $\mathrm{XX}^{\mathrm{e}}$ siècle. D'autre part se sont développées des stratégies de lecture en creux de certaines sources, notamment des sources judiciaires, pour éclairer la constitution du genre féminin, en même temps que les historien-ne-s diversifiaient les sources utilisées (histoire orale, documents audiovisuels). L'émergence de la catégorie de genre distincte a cependant suscité des critiques contre une histoire des femmes jugée trop réductrice, et a attiré l'attention sur la constitution du genre masculin, notion qui est, par un paradoxe apparent, dissimulée par l'omniprésence des hommes dans les sources. P. PASTEUR mentionna donc un certain nombre de travaux illustrant un constat déjà dressé par ailleurs, à savoir que la question est moins celle du silence des sources que du mutisme de l'historien face à ces dernières, en d'autres termes son incapacité ou sa difficulté à les « faire parler».

Dans ses remarques conclusives, Guillaume Garner (IFHA) rappela que, si les corpus de sources sont largement clos, ou si l'on postule l'absence de découverte de nouveaux documents dans le futur, les études d'histoire n'en restent pas moins perpétuellement renouvelées, puisque c'est le présent qui explique le passé et non l'inverse: par 
conséquent, les problématiques évoluent, tout comme les silences des sources, et les stratégies ou les protocoles pour surmonter ce silence. De ce point de vue, les communications font apparaitre deux cas de figure : celui d'une absence de sources, et celui de sources abondantes, voire surabondantes, mais silencieuses sur certains points. Cette journée a ainsi donné une idée de la diversité des pratiques et des stratégies pouvant être mises en œuvre pour faire face à ces deux types de silence (sachant que d'autres peuvent également être envisagées), ces procédures se fondant essentiellement sur la mobilisation d'outils théoriques empruntés à d'autres disciplines. En tout état de cause, l'approche par le silence ou par le vide a le mérite de rappeler le caractère « construit » du fait historique comme produit d'une élaboration complexe. 